\title{
Hardware-in-the-loop tuning of a feedback controller for a buck converter using a GA
}

\author{
Mr K. D. Wilkie, Dr M. P. Foster, Dr D. A. Stone and Dr C. M. Bingham \\ Department of Electronic and Electrical Engineering, The University Of Sheffield, Sheffield, S1 3JD (UK)
}

\begin{abstract}
This paper presents a methodology for tuning a PID-based feedback controller for a buck converter using the ITAE controller performance index. The controller parameters are optimized to ensure that a reasonable transient response can be achieved whilst retaining stable operation. Experimental results demonstrate the versatility of the on-line tuning methodology.
\end{abstract}

Index Terms-- DC-DC power conversion, Genetic algorithms, Proportional control, Switched mode power supplies

\section{INTRODUCTION}

For many years, PID based feedback controllers have been employed in automated control problems by virtue of their applicability to control a wide majority of dynamical systems. PID controllers are relatively easy to implement and have been applied to applications as diverse as flight surface actuation and electrical motor drive system control. Owing to these factors, many controller types have been developed, with numerous controller parameter tuning algorithms being described in the literature [1], the most popular being the ZieglerNichols (Z-N) tuning method. Although the Z-N tuning methodology provides for reasonable controller design, there are certain applications where it is impractical to determine the system responses required by the method. In these situations the 3-term PID compensator may be tuned in the following empirical manner:

i) Find an acceptable proportional gain which provides some overshoot but no sustained oscillatory behaviour.

ii) Select an integral gain that eliminates steadystate error whilst not unduly affecting stability.

iii) Finally, the addition of derivative gain to improve damping whilst not unduly increasing noise.

Typically this process results in a controller that is adequate for its purpose, but which is, in general, suboptimal in some way owing the restricted manner in which the designer chooses the PID parameters. Often the designer makes initial guesses for the controller parameter values and then adjusts these values by an order of magnitude (or by a factor of 2) until a reasonable response is obtained. Although this method can provide usable results, the plethora of values which the controller parameters can take make it nearly impossible for the designer to evaluate them all, making it highly likely that an "optimum" controller will be missed by the very coarseness of the search.

In an effort to overcome the disadvantages of classical empirical tuning techniques, this paper presents an automated method for tuning PID controller parameters using a genetic algorithm. Genetic algorithms are a convenient tool for efficiently searching non-linear workspaces where traditional gradient based methods fail or are difficult to formulate. These properties have lead to them being employed in applications as diverse as optimising order schedules for reheat furnaces [2], the design of permanent magnetic 3-phase motors [3] and the tuning of PID controller parameters for pitch-angle control of a micro air vehicles [4].

This paper utilizes similar technology to [4] for tuning a PID controller for a synchronous buck converter based on the start-up transient response. It will be shown that the proposed methodology readily determines appropriate parameter values for the PID controller.

\section{BUCK CONVERTER}

Although the buck converter is probably the simplest form of switched-mode power supply (SMPS) circuit, it is still widely used today for efficiently reducing a highvoltage to a lower voltage level more suitable for the intended application. Fig. 1 shows the basic circuit for a buck converter.

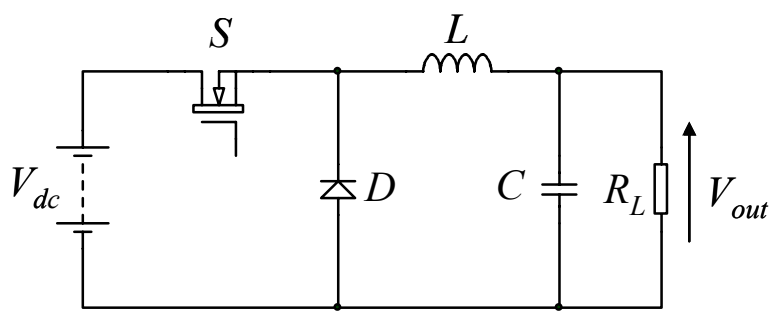

Fig. 1 Buck converter

Operation of the circuit is as follows: the DC input voltage $V_{d c}$ is chopped at high-frequency by switching leg $S \& D$ to form a rectangular shape voltage waveform. This high-frequency rectangular waveform is then passed through the low-pass filter, formed by $L \& C$, which attenuates the high-frequency components of the voltage essentially leaving a DC component (the average value) which is taken to be the output voltage $V_{\text {out }}$ of the SMPS. 
$V_{\text {out }}$ is controlled by varying the on-to-off ( $t_{o n}$-to- $\left.t_{\text {off }}\right)$ time of the switching leg. Analysis presented in many texts, [5] for example, shows that the output voltage is proportional to the switch on-time to switching period ratio and the DC input voltage,

$$
V_{o u t} \propto \frac{t_{o n}}{T} V_{d c}
$$

where the switching period $T=t_{\text {on }}+t_{\text {off }}$.

The output voltage can easily be controlled by varying duty cycle $\left(d=t_{o n} / T\right)$ of $S$ such that $V_{\text {out }}$ is maintained at a steady value despite of changes in the input voltage and load. Many control methodologies for the buck converter have been proposed but it is usual to regulate the output voltage directly using voltage-mode control or to regulate the output voltage indirectly using current-mode control [5]. The work presented in this paper concentrates on voltage-mode control because of its simplicity of implementation, however, the findings are also applicable to current-mode control and other more esoteric control techniques.

\section{PID CONTROLLER}

In order for the output voltage to remain at a stable value it is necessary to compare its value to a reference value $V_{\text {ref }}$ (sometimes called a demand voltage) and then take appropriate remedial action to ensure that $V_{\text {out }}=V_{\text {ref }}$. Usually, this is achieved by generating an error signal $e=V_{\text {out }}-V_{\text {ref }}$ which the controller (sometimes referred to as a compensator) then manipulates in such a manner so as to adjust $V_{\text {out }}$ to ensure it is always equal to the reference. The three-term PID controller, which is the focus of this paper, is one example of a wide variety of controllers that are suitable as compensators to counteract variations in input voltage or load.

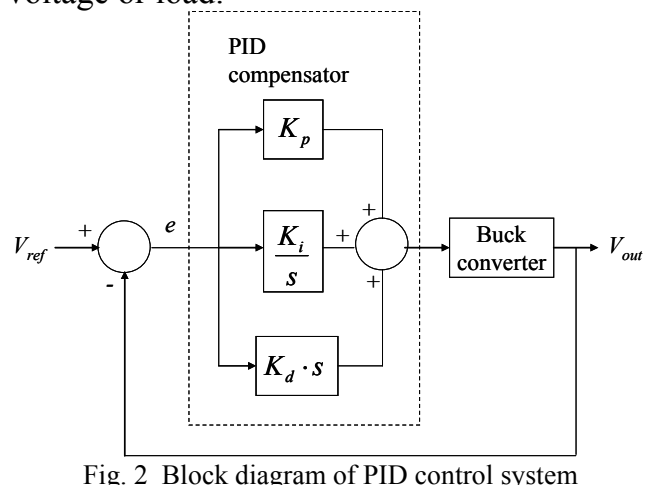

Referring to Fig. 2, the output voltage is compared to a reference producing an error signal, $e$. The error signal is individually applied to each term of the compensator after which they are combined forming the duty cycle input command to the buck converter. The proportional gain $K_{p}$ acts as a feed-forward term allowing any changes in the error to be passed to the compensator output without delay. $K_{p}$ must be carefully chosen because large values tend to induce instabilities in the system response. The integral term $K_{i}$ is used to reduce the steady-state error at the expense of reducing the dynamic response. Finally, the derivative term $K_{d}$ is employed to improve the dynamic response.

The performance of each prototype controller was evaluated using the ITAE (Integral of time multiplied by absolute error) performance index [1]. The ITAE index (2) evaluates a controller's performance by assigning it a score based on the error response of the system. Essentially, good performing controllers will have lower ITAE scores than poor performing counterparts. As can be appreciated, the error signal could possibly have a large value the instant in time after a transient event. To ensure that this phenomenon does not unduly affect a controller's score, the absolute error is multiplied by time (which should be a small value in the vicinity of the transient event) to reduce its impact.

$$
I T A E=\int t|e| d t
$$

\section{GENETIC ALGORITHM}

Genetic algorithms borrow ideas from evolution theory to intelligently explore a parameter space while optimizing a function. The problem is broken down into a 'population' of candidate solutions which are refined over a number of 'generations'. A single candidate solution is represented by a 'chromosome' which essentially encodes all of the optimizable parameters into a single entity. Each candidate is ranked using a fitness function (here, this is the ITAE performance index) and those with the best fitness score are selected for further refinement. The refinement stage then operates on each of the chromosomes by i) 'breeding' - a process where a new population of improved candidates are generated using the present generation's population, and ii) 'mutation' - in which chromosomes are modified in some way. Both of these operations to permit the parameter space to be more effectively explored. The whole process is iterated for many generations where the candidate solutions can be seen to evolve and, hopefully, converge towards a single solution. Fig. 3 diagrammatically represents the major stages of GA optimization.

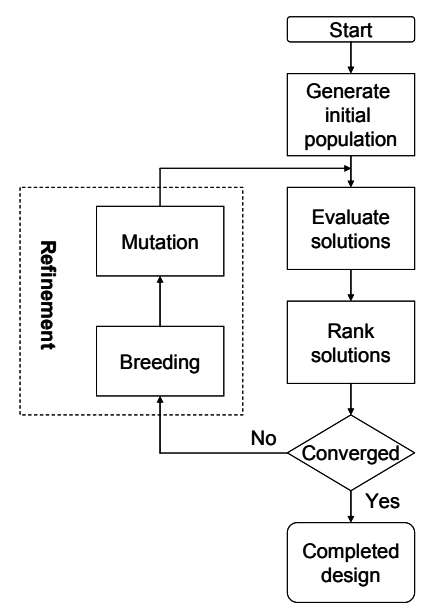

Fig. 3 Flowchart of a typical GA 
Further details regarding genetic algorithms and their implementation can be found in [6].

\section{IMPLEMENTATION DETAILS}

As is evident from the preceding sections, the whole buck converter tuning system contains three main subsystems: i) the buck converter, ii) the controller and iii) the genetic algorithm which was implemented in Matlab on a PC. The interaction of each sub-system is represented in Fig 4.

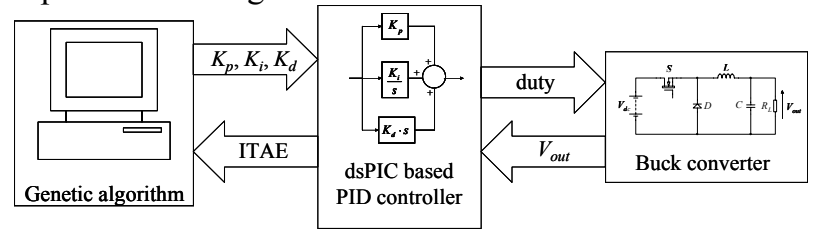

Fig. 4 Block diagram of tuning system

\section{A. Buck converter}

A buck converter was implemented using the parameters detailed in Table I. It should be noted that the authors felt it necessary to replace the 'free wheeling' diode $D$ with a MOSFET for two reasons: i) most commercial buck converters use a synchronous rectifier to increase efficiency, and ii) the synchronous rectifier permits negative inductor current which means that the prototype should always exhibit continuous current mode control characteristics for the majority of its operating range.

TABLE I

PROTOTYPE CONVERTER PARAMETERS

\begin{tabular}{|c|c|c|c|}
\hline $\mathrm{L}$ & $\mathrm{C}$ & $\mathrm{R}$ & $\mathrm{f}_{\mathrm{PWM}}$ \\
\hline $1.14 \mathrm{mH}$ & $4.7 \mu \mathrm{F}$ & $22 \Omega$ & $100 \mathrm{kHz}$ \\
\hline
\end{tabular}

\section{B. Controller}

The feedback controller was implemented on a Microchip dsPIC30F4011 high-performance microcontroller. The dsPIC30F4011 is specifically designed for switched-mode power supply and motor control applications and features high-accuracy PWM generation units, 10-bit ADCs which can be sampled at $100 \mathrm{k}$ samples per second and a modified ALU with a built-in hardware multiply-accumulator (MAC) enabling filter and controller algorithms to be implemented with much reduced loop times when compared to traditional non-MAC cores.

In this application the dsPIC had the task of i) receiving controller parameters from the host $\mathrm{PC}$, ii) exercising the buck converter with a startup transient and attributing a score to the parameters, and iii) communicating the score back to the host PC. The controller had a loop time of $45 \mu \mathrm{s}$.

Regarding the control algorithm, Microchip has kindly included a suite of PID control related functions as part of their C-language compiler [7]. Since these function utilize the MAC, a single control output can be evaluated in microseconds depending on the oscillator frequency. Microchip's particular implementation of the PID control algorithm uses a fractional integer representation for the parameter values which significantly simplified the design of the GA.

\section{Genetic algorithm}

Although the dsPIC uses a fraction integer representation, a floating point (or continuous variable) representation for the PID parameters chosen because of the peculiarities associated with Microchip's implementation of the PID control algorithm [6]. Each chromosome was represented by an array of three values, each value being associated with the controller parameters $K_{p}, K_{i}$ and $K_{d}$. The dsPIC then evaluates the performance of a candidate controller and returns an ITAE scores. The ITAE scores are ranked in ascending order, the best controller having the lowest ITAE score. The refinement stage then determines appropriate controller parameter values for the next generation.

The refinement stage involves the selection of suitable parent chromosomes from which an offspring can be obtained using a breeding process. Tournament selection is used to obtain the parents from which the two children are derived. Two parent chromosomes are selected at random and the one with the highest ranking is chosen to be parent $p_{l}$. Another two parents are selected at random from which parent $p_{2}$ is obtained. The children $c_{1}$ and $c_{2}$ are obtained by combining the parents in a linear manner. If $p_{1}$ and $p_{2}$ are parent chromosome parents, then their contributions to the children $c_{1}$ and $c_{2}$ are determined by a random number $\alpha$ as follows,

$$
\begin{aligned}
& c_{1}=\alpha p_{1}+(1-\alpha) p_{2} \\
& c_{2}=(1-\alpha) p_{1}+\alpha p_{2}
\end{aligned}
$$

where $\alpha$ is randomly generated number between 0 and 1 .

In addition, to ensure there is consistency between subsequent generations an elitism strategy is adopted where $K$ highly ranked chromosomes from a total population of $P$ chromosomes would be retained for the next generation, with the remaining $P-K$ chromosomes being replaced via the breeding process. In this application the authors decided to keep $50 \%$ of the population (i.e. $K=0.5$ ). All the results presented in this paper used a population of 20 chromosomes.

The mutation process involved randomly selecting a parameter value from the entire chromosome population and then replacing it with a random value. The mutation rate was set at $50 \%$ to ensure the search space was adequately explored. In order to ensure the best characteristics were inherited from previous generations, the highest ranking chromosome is excluded from the mutation process.

For the first generation, one chromosome in the population is initialized by the user to give the GA a good starting point. The remaining chromosomes were initialized using a random number generator.

\section{RESULTS}

With the reference voltage set at $5 \mathrm{~V}$ and the input voltage set to $12 \mathrm{~V}$ the PID controller was manually tuned to obtain a reasonable transient response. The following parameters $\left(K_{p}=0.1, K_{i}=0.01, K_{d}=0\right)$ provided an ITAE 
score of 207022 and gave the startup transient response shown in Fig. 5a. The GA was initialized with these starting values and run for 20 generations. The highest ranking candidate, after ITAE optimization, had the parameter values $\left(K_{p}=0.6797, K_{i}=0.6518, K_{d}=0.789\right)$, an ITAE $=23154$ and gave the transient response of Fig. $5 \mathrm{~b}$. As can be seen, by virtue of its reduced overshoot and settling time the ITAE optimized controller provides a superior transient response. Indeed, the output voltage settling time has been reduced from $4 \mathrm{~ms}$ to $0.7 \mathrm{~ms}$

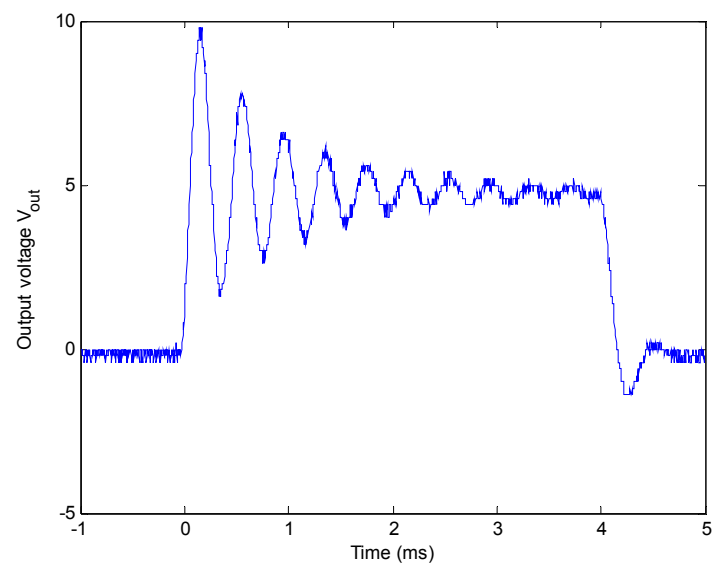

(a)

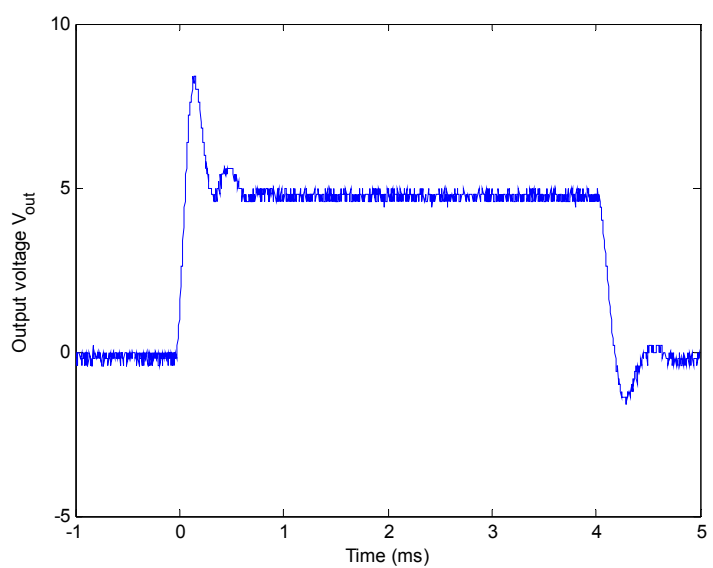

(b)

Fig. 5 Startup transient response of the buck converter with $V_{\text {ref }}=5 \mathrm{~V} \&$ $V_{d c}=12 \mathrm{~V}$. a) Manual tuned, b) GA tuned

Fig. 6 shows the parameter search space $\left(K_{p}-K_{i}\right)$ and $\left(K_{p}-K_{d}\right)$ as a function of ITAE. The figure clearly illustrates the searching abilities of the GA owing to the wide distribution of evaluations distributed throughout the search space. It is interesting to note that the GA conducted a detailed search of the parameter space associated with $K_{p}$ values between 0.6 and 0.7 , particularly evident in Fig. 6b. Indeed, constraining the data plot to show ITAE values less than the population mean as depicted in Fig. $7 \mathrm{~b}$ shows that the GA considered the range of values $0.6 \leq K_{p} \leq 0.7$ to be of considerable interest.

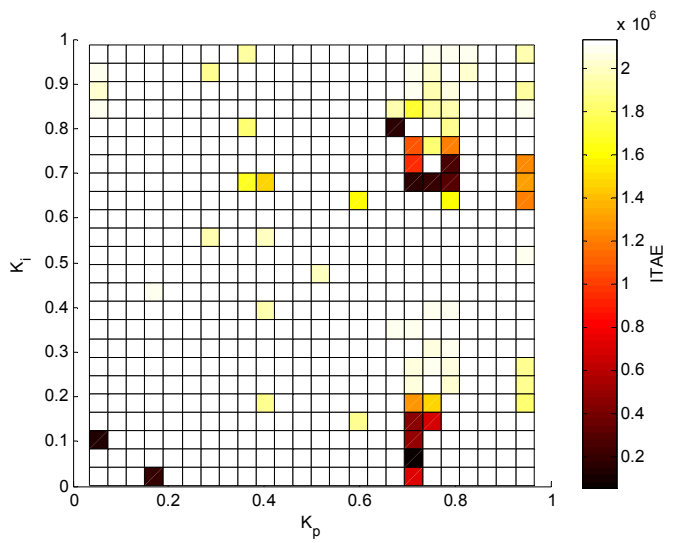

(a)

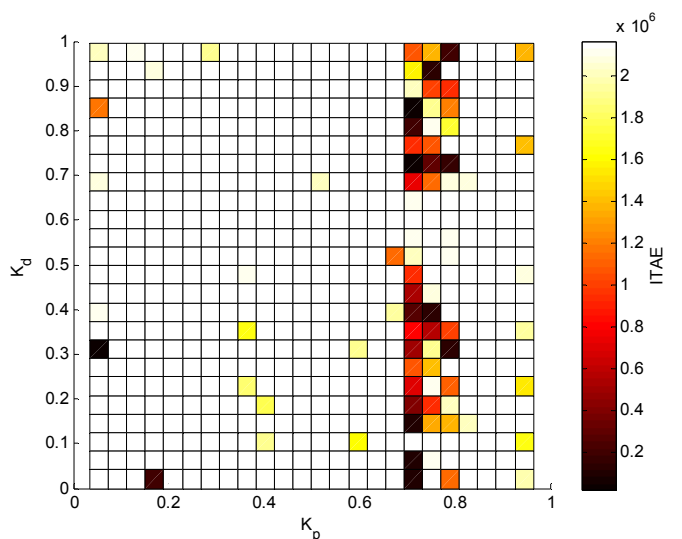

(b)

Fig. 6 PID controller parameters as a function of ITAE

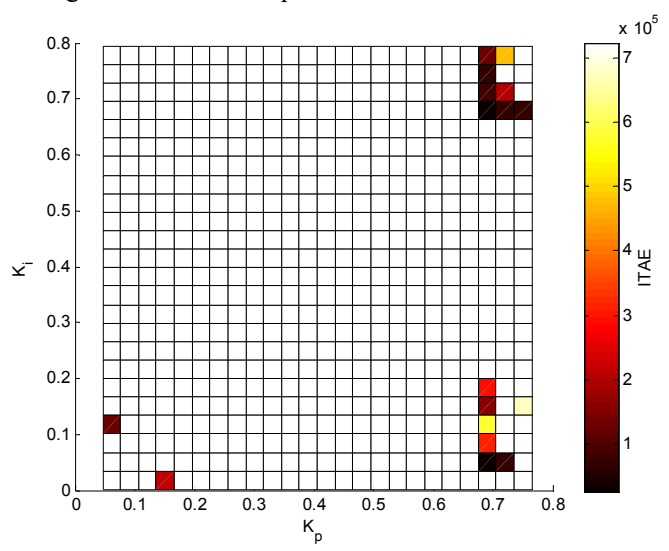

(a)

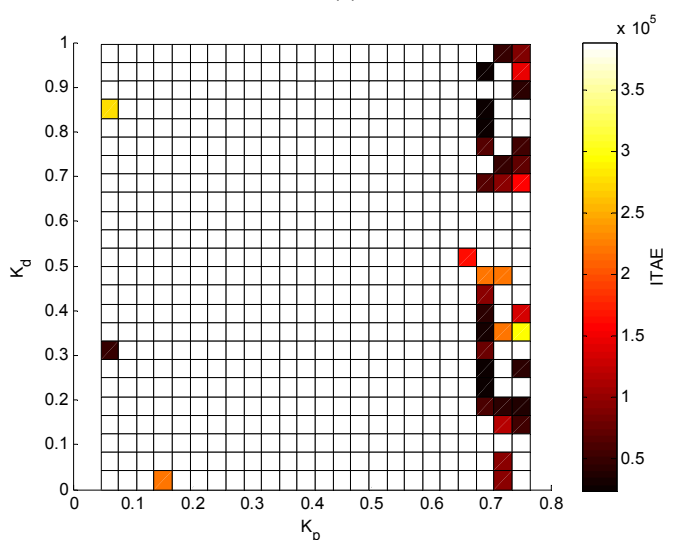

(b)

Fig. 7 PID controller parameters as a function of ITAE with ITAE less than mean ITAE 
The convergence properties of the GA are illustrated in Fig. 8 where it can be seen that the average ITAE value per generation steadily improves as the population converges towards an optimized solution. The best ITAE value associated with a particular generation also indicates this trend. Usually one would expect the best ITAE score to decrease monotonically with increasing generations, however, in this application the best ITAE score is seen to both increase and decrease in value from one generation to the next. The reason for this is that the measurements were performed using hardware-in-theloop which means signal measurements are subject to noise and other real-world influences such as heating effects. Although not attempted here, the influence of these phenomena of tuning performance could be reduced by taking the mean value of several runs.
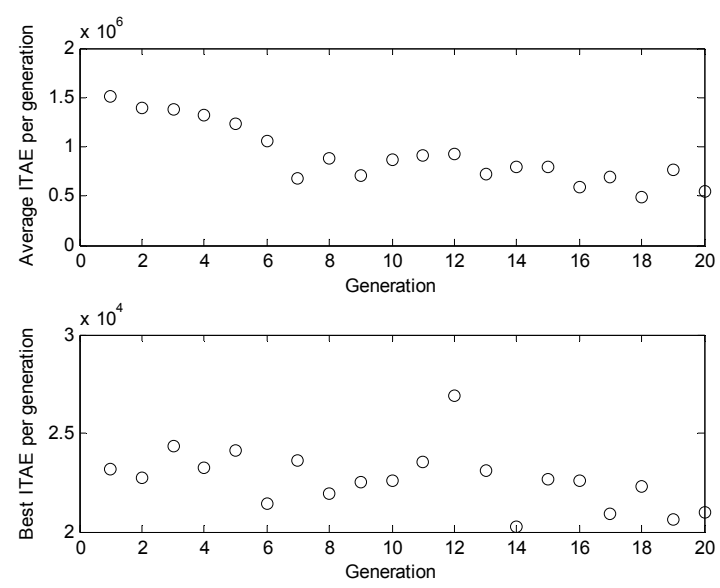

Fig. 8 Convergence properties of the proposed genetic algorithm

In an effort to reduce the overshoot in the output voltage a new cost function $J$ was devised which included a term penalize large overshoot values. Scaling the original ITAE score and including an extra term featuring the maximum output voltage $V_{\text {out_max }}$ and reference voltage $V_{\text {ref }}$ gave,

$$
J=\frac{I T A E}{100000}+10 \times\left|\frac{V_{\text {out_max }}-V_{\text {ref }}}{V_{\text {ref }}}\right|
$$

The initial controller gave a score of $J=218155$. The GA was once again run for 20 generations which provided new controller parameter values of $\left(K_{p}=0.6697\right.$, $\left.K_{i}=0.0204, K_{d}=0.3859\right)$ with a score $J=20282$. The output voltage startup transient obtained with the new controller parameters, Fig. 9, shows the maximum overshoot voltage has been reduced from $8.4 \mathrm{~V}$ (Fig. 5b) to $7.4 \mathrm{~V}$. This overshoot could be reduced even further by altering the weightings on the ITAE and overshoot terms in (4).

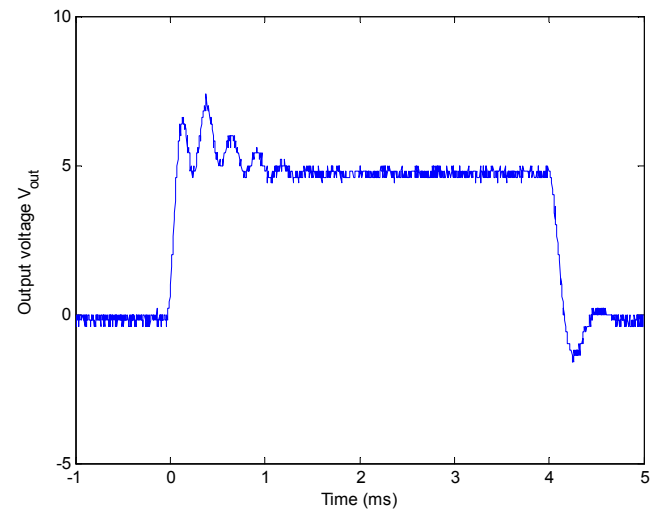

Fig. 9 Startup transient response from PID controller parameters obtained using the overshoot reduction cost function

\section{CONCLUSIONS}

An automated tuning methodology for optimizing PID controller parameter values regulating the output voltage of buck converter has been described. The performance of a given controller was evaluated using the ITAE performance index criterion. A genetic algorithm was used to search the PID parameter space to minimize the ITAE index. Experimental tests have demonstrated that the GA can readily provide improved transient responses in comparison to manual heuristic tuning methods. Implementation details regarding the controller and genetic algorithm have been provided.

\section{REFERENCES}

[1] Atröm, K. J. \& Hägglund, T.; 'PID Controllers: Theory, Design and Tuning', International Society for Measurement and Control, 1995.

[2] Broughton, J., Mahfouf, M., Linkens, D. A.; 'GA-Based Optimisation of a Continuous Walking Beam Reheating Furnace', UKCI 2003 The 2003 UK Workshop on Computational Intelligence, CD-ROM proceedings.

[3] Bianchi, N. and Bolognani, S.; 'Design optimisation of electric motors by genetic algorithms', IEE Proc. Electric Power Applications, September 1998, Vol. 145, Iss. 5, p. 475-483.

[4] Essex, N. C., Foster, M. P., Bingham, C. M. \& Kuo, C.; 'GA tuning of Pitch Controller for Small Scale MAVs', World Scientific and Engineering Academy and Society (WSEAS) World Conference, Cairo - Egypt, December 2007.

[5] Mohan, N., Underland, T. M., and Robbins, W. P.; 'Power Electronics: Converters, Applications and Design', John Wiley \& Sons.

[6] Goldberg, D. E.; 'Genetic Algorithms in Search, Optimization, and Machine Learning', Addison-Wesley Professional.

[7] DS51456D - 16-Bit Language Tools Libraries, Microchip 